Sammlung Metzler

Band 312 
Wolfgang Albrecht

\section{Arno Schmidt}

Verlag J.B. Metzler

Stuttgart - Weimar 
Die Deutsche Bibliothek - CIP-Einheitsaufnahme

Albrecht, Wolfgang:

Arno Schmidt / Wolfgang Albrecht.

- Stuttgart ; Weimar : Metzler, 1998

(Sammlung Metzler ; Bd. 312)

ISBN 978-3-476-10312-3

ISBN 978-3-476-10312-3

ISBN 978-3-476-05160-8 (eBook)

DOI 10.1007/978-3-476-05160-8

ISSN 05583667

\section{SM 312}

Dieses Werk einschließlich aller seiner Teile ist urheberrechtlich geschützt. Jede Verwertung außerhalb der engen Grenzen des Urheberrechtsgesetzes ist ohne Zustimmung des Verlages unzulässig und strafbar. Das gilt insbesondere für Vervielfältigungen, Übersetzungen, Mikroverfilmungen und die Einspeicherung und Verarbeitung in elektronischen Systemen.

(C) 1998 Springer-Verlag GmbH Deutschland

Ursprünglich erschienen bei J.B. Metzlersche Verlagsbuchhandlung und Carl Ernst Poeschel Verlag GmbH in Stuttgart 1998 


\section{Inhalt}

Abkürzungen und Siglen $\ldots \ldots \ldots \ldots \ldots \ldots \ldots \ldots$ VII

1. Leben und Werk -

Überleben in und durch Dichtung ............ 1

1.1 Fremdbestimmtheit und Selbstsuche:

Schüler und Autodidakt, schriftstellerische Anfänge

als Angesteller und Soldat

(Hamburg, Schlesien, Norwegen 1914-1945) ....... 2

1.1 .1 Juvenilia ........................ 6

1.1.2 Dichtergespräche im Elysium .............. 7

1.1.3 Pharos oder von der Macht der Dichter.......... 8

1.2 Drangvolle Selbstfindung: Unstete Umsiedler-

und Schriftstellerexistenz

(Cordingen, Gau-Bickelheim, Kastel 1945-1955) ... 13

1.2.1 Leviathan oder Die beste der Welten .......... 15

1.2 .2 Antike-Erzählungen................... 20

1.2.3 Aus dem Leben eines Fauns ................ 27

1.2.4 Warnutopien und Utopieparodien........... 32

1.2.5 Seelandschaft mit Pocahontas ................ 39

1.3 Zwischenstation: "Darmstadt i. d. Barbarei«

(1955-1958) ...................... 43

1.3.1 Das steinerne Herz ................. 43

1.3.2 Fouqué und einige seiner Zeitgenossen ........ 49

1.3.3 Texte für Presse und Rundfunk; Übersetzungen ... . 52

1.4 Entfesselte Selbstentfaltung: Im Bargfelder Refugium

(1958-1979) ........................ 56

1.4.1 Kaff auch Mare Crisium ............... 58

1.4 .2 Kühe in Halbtrauer . . . . . . . . . . . . . . . 66

1.4 .3 Zettels Traum ..................... 71

1.4.4 Die Schule der Atheisten . . . . . . . . . . . . . . 83

1.4 .5 Abend mit Goldrand .................. 91

\section{Arno Schmidts Prosadichtung -}

Selbstansichten und Forschungsbefunde ....... 98

2.1 Realismus und Phantasie ................ 99

2.2 Handlungsstrukturen und Erzählformen ......... 102

2.3 Sprach- und Zitierkunst zwischen Poetisierung und Verschlüsselung; Plagiats-Probleme ......... 105 
2.4 Wirkungsstrategien, Rezeptionsvorgaben und Rezeptionsbarrieren ... . . . . . . . . . . . . . 109

2.5 Avantgardismus und Traditionalismus;

Modernitäts-Problem ................... 113

3. Abriß zur Wirkungsgeschichte........... 116

3.1 Resonanz zu Lebzeiten ................ 118

3.2 Wachsender Nachruhm ................ 123

3.3 Hauptrichtungen und Tendenzen der Forschung . . . 128

4. Grundlageninformationen ............. 132

4.1 Editionen und Nachlaß (vgl. Kap. 5.1) . . . . . . 133

4.2 Bibliographien und Literaturberichte

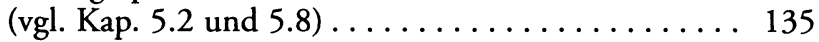

4.3 Biographien und Dokumentationen, Kataloge und Kommentarbände (vgl. Kap. 5.2-3) . . . . . . 137

5. Auswahlbibliographie ............... 140

5.1 Editionen, Einzelausgaben, Faksimiledrucke ...... 141

5.2 Bibliographien, Lebens- und Tondokumente ....... 142

5.3 Zu Leben und Werk insgesamt und zu einzelnen Perioden ........................ 143

5.4 Persönliche und literarische Beziehungen ........ 145

5.5 Sammel- und Konferenzbände . . . . . . . . . . . 147

5.6 Thematische Untersuchungen . . . . . . . . . . 147

$5.7 \mathrm{Zu}$ Einzelwerken.................... 151 Übersicht . . . . . . . . . . . . . . . . 151

5.8 Rezeptions-, Wirkungs- und Forschungsgeschichte .. 162

5.9 Index. . . . . . . . . . . . . . . . . . . . 164

6. Anhang ....................... 169

6.1 Register der Werke Schmidts . . . . . . . . . . . . 169

6.2 Personenregister .................. 170

Angaben zum Autor .................... 175 


\section{Abkürzungen und Siglen}

(Bei Siglen angefügte römische und arabische Ziffern bezeichnen die Band- bzw. Seitenzahlen; Ausnahme: Bargfelder Ausgabe, s. Sigle BA.)

AS

Aufl.

BA

BB

Bd.

Dass.

Diss.

DVjs

e.

ED

EDW

EE

H.

Hab.schr.

$\mathrm{HB}$

Hg./hg. $\mathrm{Hs}(\mathrm{s})$.

$\mathrm{Jb}(\mathrm{b})$.

Jg.

Jh.

Lfg.

Ma.

N.F.

$\mathrm{Nr}$.

s.

S.

s.a.

ÜAS I,II udT

Arno Schmidt (nur in der Auswahlbibliographie)

Auflage

Arno Schmidt: Werke. Bargfelder Ausgabe (s. Nr. 1; bei

Zitatnachweisen mit römischer Abteilungs- und arabischer Bandzahl angegeben)

Bargfelder Bote. Materialien zum Werk AS. Hg. v. Jörg Drews. München 1972ff. (erschient in Lieferungen; bis September 1997: Lfg. 219-221)

Band

Dasselbe

Dissertation

Deutsche Vierteljahrsschrift für Literaturwissenschaft und Geistesgeschichte

entstanden

Erstdruck

Entstehungs-, druck- und wirkungsgeschichtliche Darlegungen

Einzelstellenerläuterungen, -entschlüsselungen

Heft

Habilitationsschrift

Handbuch (zu einem bestimmten Einzelwerk Schmidts)

Herausgeber / herausgegeben

Handschrift(en)

Jahrbuch (Jahrbücher)

Jahrgang

Jahrhundert

Lieferung

Magisterarbeit

Neue Folge

Nummer (in der Auswahlbibliographie im vorliegenden Band)

siehe

Seite

siehe auch

Über Arno Schmidt (s. Nr. 287f.)

unter dem Titel 
unpag. unpaginiert

u.ö. und öfter

verb. verbessert

VzZT Arno Schmidt: Vorläufiges zu Zettels Traum (s. Nr. 25)

ZK Zettelkasten. Aufsätze und Arbeiten zum Werk AS. Jb. der Gesellschaft der AS-Leser

Zs. Zeitschrift

ZT Arno Schmidt: Zettels Traum (s. Nr. 8), 4. Aufl. 1986 